\title{
Residual Noise Identification for PIM Measurement Systems using Variable Low-PIM Termination
}

This paper was downloaded from TechRxiv (https://www.techrxiv.org).

\section{LICENSE}

CC BY 4.0

SUBMISSION DATE / POSTED DATE

04-01-2022 / 07-01-2022

\section{CITATION}

Kuwata, Masayoshi; Yamashita, Tamon; kuga, Nobuhiro (2022): Residual Noise Identification for PIM Measurement Systems using Variable Low-PIM Termination. TechRxiv. Preprint. https://doi.org/10.36227/techrxiv.17793842.v1

$\mathrm{DOI}$ 


\title{
Residual Noise Identification for PIM Measurement Systems using Variable Low-PIM Termination
}

\author{
Masayoshi Kuwata, Student Member, IEEE, Tamon Yamashita, Student Member, IEEE, \\ and Nobuhiro Kuga, Member, IEEE
}

\begin{abstract}
In this letter, we describe the design for the variable low-PIM termination composed of the voltage-controlled IMsource, a fixed attenuator, and a linear attenuator. The design method for evaluating PIM-level of the fixed attenuator is presented in order not to limit the variable range of entire termination. It is possible to maintain low-PIM performance in spite of using active voltage-controlled IM-source, whose IM level is extremely high. This termination is used for a dynamic residual noise identification for PIM measurement systems by observing the saturation value for voltage sweep. The validity is confirmed by experiments in $2 \mathrm{GHz}$ band.
\end{abstract}

Index Terms-nonlinear noise, passive intermodulation, residual noise, diode

\section{INTRODUCTION}

Intermodulation (IM) caused by non-linearity in RF devices is unwanted wave which may degrade the quality of communication system. In particular, the one generated by the passive element or circuit is called PIM (Passive IM). PIM is often ignored because of whose weak power compared with transmitting signals. However, it may become unexpected interference in wireless communication systems where downlink power is much stronger than up-link [1]-[4]. Therefore, PIM performance for RF components should be carefully evaluated.

In general, low-PIM characteristics are required for the PIM measurement system (PIM tester) [6]. Unwanted PIM generated inside of measurement system are called system residual noise. In order to evaluate the PIM characteristics of the device under test (DUT) with high accuracy, it is necessary to confirm that the system residual noise is sufficiently reduced from the desired measurement level.

PIM measurement is commonly performed with DUT terminated in good impedance matching condition. Since careful evaluation for residual noise in good matching condition is desired, appropriate termination with low-PIM performance (low-PIM termination) is required. PIM characteristics of resistive components has been investigated [5], and two major low-PIM termination have been proposed so far [6], [7]. The first one uses a long low-loss transmission line to absorb power sufficiently [6]. In this letter, it is referred as a long-line

Masayoshi Kuwata and Nobuhiro Kuga are with the Graduate School of Engineering Science and Tamon Yamashita is with Interfaculty Graduate School,Yokohama National University, 79-5 Tokiwadai, Hodogaya-ku, Yokohama-shi, 240-8501, Japan, e-mail: (kuwata-masayoshi-yv@ynu.jp)

Manuscript received April 19, 2005; revised August 26, 2015.
low-PIM termination. It has well known that lossy materials tend to exhibit non-linear behavior, which leads to high-PIM performance. Hence, the one with a long low-loss transmission line realizes low-PIM performance [5]. However, it has the disadvantage of requiring extremely large size with respect to the wavelength. The second one is that the edge of the long-line low-PIM termination is replaced with a resistive termination [7]. In this letter, it is refered as a resistive lowPIM termination. In this design, power absorption is mainly performed by the resistive termination, and its generated large PIM is reduced to the desired PIM level by adjusting the length of the low-loss transmission line connected to the resistive termination. Therefore, it is possible to shorten whole size compared with long-line low-PIM termination.

The above two low-PIM terminations are widely used for PIM measurement. Since quantitative PIM evaluation for these terminations are difficult, it is desirable to measure the performance experimentally before the test. However, in order to evaluate the characteristics of these termination, a highperformance PIM tester with lower system residual noise is required. Therefore, it is difficult to evaluate the performance of PIM testers and the low PIM termination at the same time.

From this background, a novel termination which enables to vary PIM performance is required. For example, by varying PIM level, if it saturates to a certain value, it is confirmed as the PIM level inside the system. Otherwise, observed PIM level is the one caused by the termination. In this way, the source of PIM can be easily clarified. Thus, this method enables to dynamically identify residual noise from the saturation value with good stability.

In this letter, we propose variable low-PIM termination which has voltage-controlled IM generator. Its variable IMsource consists of a schotkky diode is installed in proposed termination, and the amount of generated PIM can be controlled by the bias voltage. In addition, dynamic identification method for residual noise is also proposed. Validity is confirmed through 2-tone test for the 3rd-order PIM in $2 \mathrm{GHz}$ band. In this study, the third-order PIM $f_{\text {pim }}=2 f_{1}-f_{2}=1.90 \mathrm{GHz}$ for $f_{1}=2.05 \mathrm{GHz}$ and $f_{2}=2.20 \mathrm{GHz}$ is observed.

\section{THE DESIGN METHOD FOR VARIABLE LOW-PIM TERMINATION}

Fig. 1 (a) shows the configuration of the variable low-PIM termination proposed herein. This termination is divided into 
three parts: linear attenuator, fixed attenuator, and variable IM-source. Linear attenuator is a long low-loss transmission line made from UT141 which is identical to that used in the literature [7]. It attenuates the input power sufficiently with maintaining low-PIM performance. In order to suppress the PIM generated from the connector, both edges are made with 7/16-DIN connectors [8]. A SMA-type 20dB fixed attenuator (Orient Microwave BX20- 0476-00) is employed as the fixed attenuator, which produces relatively high PIM [8]. Since fixed attenuator should be connected to linear attenuator with maintaining low PIM-performance, it is connected via a commercially low-PIM adapter (Pasternack PE91036). Variable IM-source is composed of bias tea, IM source, and resistive termination. Fig. 1(b) shows the configuration of the IM source. A commercially available Schottky diode (Rohm RB$721 \mathrm{Q}$ ) is loaded at center slit of the $50 \Omega$ microstrip line. The dielectric substrate with a thickness of $h=1.6 \mathrm{~mm}$ and a relative permittivity of $\epsilon_{r}=2.6$ is used herein. DC bias voltage $E[\mathrm{~V}]$ is applied to this IM source by a variable voltage source via a bias tee.

Fig. 2 shows the PIM characteristics of the variable IMsource for the bias voltage, where the variable IM-source is directly connected to PIM tester and measured. The input power is $P_{\text {in }}=3[\mathrm{dBm} /$ tone $]$, and a popular SMA-type termination is employed here. It is confirmed that the proposed IM-source produces voltage-controlled IM in about 30dB.
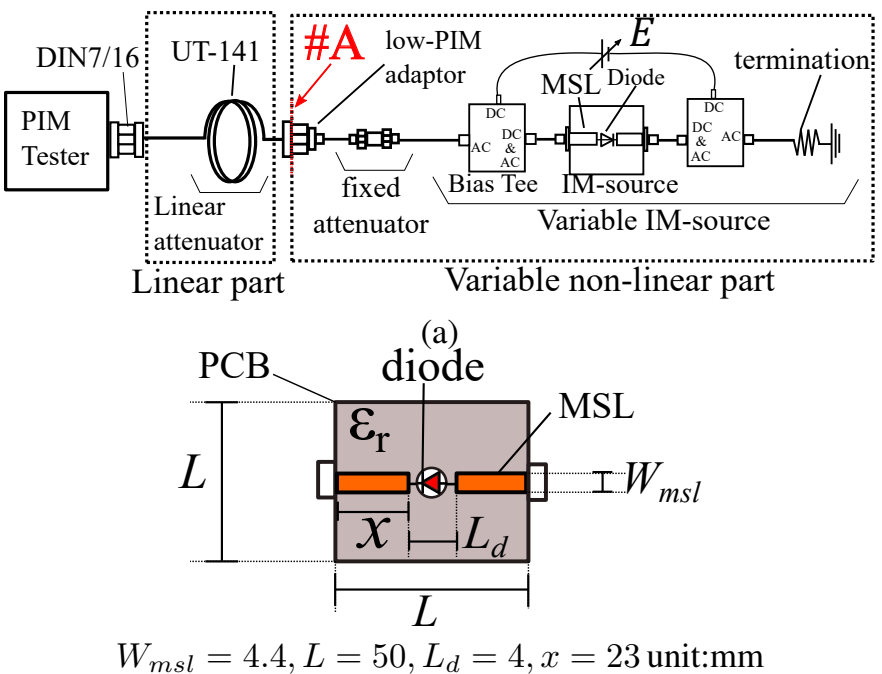

(b)

Fig. 1. (a)Configuration of variable low-PIM termination. (b)Configuration of IM-source consisted of schotkky diode

The PIM level generated by the linear attenuator is very small. Thus, this configuration is divided into two part; linear part where PIM is not produced (linear attenuator) and variable non-linear part where PIM is produced (fixed attenuator and variable IM-source). The PIM level caused by the variable non-linear part $P_{v a r}$ is expressed by the following equation.

$$
P_{\text {var }}=10 \log _{10}\left|10^{\frac{P_{d i o}+C\left(P_{i n}^{\prime}+X-P_{i n}\right)+X}{10}}+10^{\frac{P_{0 a}}{10}} e^{-j \delta}\right|
$$

$P_{0 a}[\mathrm{dBm}]$ is the PIM level generated by the fixed attenuator, and $X[\mathrm{~dB}]$ is its attenuation. In this study, we used fixed attenuation with $X=-20 \mathrm{~dB} . P_{\text {dio }}$ is the PIM level generated

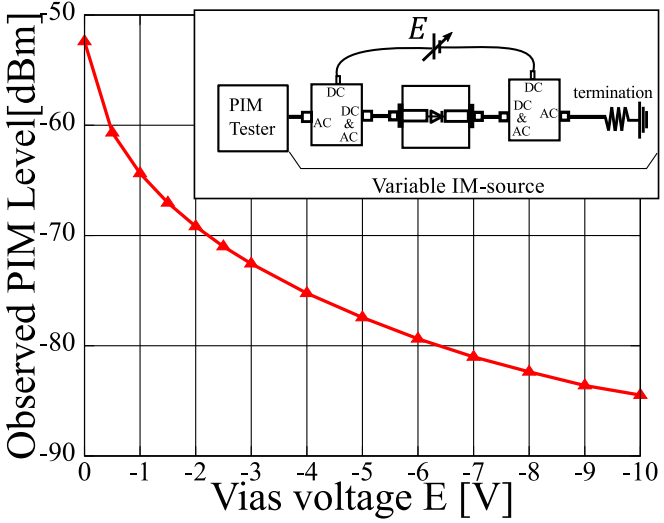

Fig. 2. PIM characteristics of IM source for reverse bias voltage $\left(P_{i n}=\right.$ $3 \mathrm{dBm} /$ tone)

by the variable IM-source which is a function of vias voltage $E$ [V]. $C$ is increment coefficient of the PIM level generated by IM-source. $C$ is commonly referred as power slope, and it is generally known that $C=3$ when observing a thirdorder PIM. Since the diode is a non-linear element producing strong IM, the PIM level generated by the passive device such as the termination and the bias tees can be ignored. Note that $\delta$ indicates the phase difference between the IM generated by the variable IM-source and the PIM generated by the fixed attenuator. As we can see from this equation, the whole PIM level generated by the variable non-linear part becomes constant value as the attenuation of the fixed attenuator $X[\mathrm{~dB}]$ increases or the PIM level generated by the fixed attenuator $P_{0 a}$ increases. Therefore, it is necessary to select the appropriate fixed attenuator and evaluate a variable non-linear part as a whole so that a sufficient variable range should be assured in residual noise identification described later.

Fig. 3 shows the PIM level generated by the variable nonlinear part for the reverse bias voltage. In this case, PIM tester is connected to \#A in Fig. 1(a). The input power is $P_{i n}^{\prime}=23$ $[\mathrm{dBm} / \mathrm{tone}]$. The dotted line and the dash-dot line show the calculated values in the equation (1) where the PIM level generated by the fixed attenuation is $P_{0 a}=-\infty,-100,-90 \mathrm{dBm}$. In this calculation, phase difference is assumed to be $\delta=0 \mathrm{deg}$. The PIM level $P_{d i o}$ and the input power of the variable IMsource $P_{i n}$ is based on the result of Fig. 2. It is confirmed that the variable range is limited by the PIM level of the fixed attenuator $P_{0 a}$. However, since it is variable by about $25 \mathrm{~dB}$ in spite of installing fixed attenuator with SMA connector, it has sufficient performance for residual noise identification described later.

As mentioned above, linear attenuator has low-PIM performance. Thus, the PIM level generated by the whole variable low-PIM termination is expressed by the following equation.

$$
P_{\text {ter }}=3\left(P_{i n}^{\prime \prime}-P_{i n}^{\prime}\right)+4 S_{L}+P_{\text {var }}
$$

$P_{i n}^{\prime \prime}$ is the input power, and $S_{L}[\mathrm{~dB}]$ is the attenuation of the linear attenuator. The PIM level of the entire variable low-PIM termination $P_{t e r}$ can be varied by the bias voltage $E[\mathrm{~V}]$. Then, the maximum value of $P_{t e r}$ is denoted as $P_{\text {ter, } \max }$ and the 
minimum value is denoted as $P_{\text {ter.min }}$. Thus, the following condition should be satisfied if desired residual noise Pst is given to be identified.

$$
P_{\text {ter, } \max }<P_{\text {st }}<P_{\text {ter, } \text { min }}
$$

Therefore, the linear attenuator should be designed so that $S_{L}$ satisfies this equation.

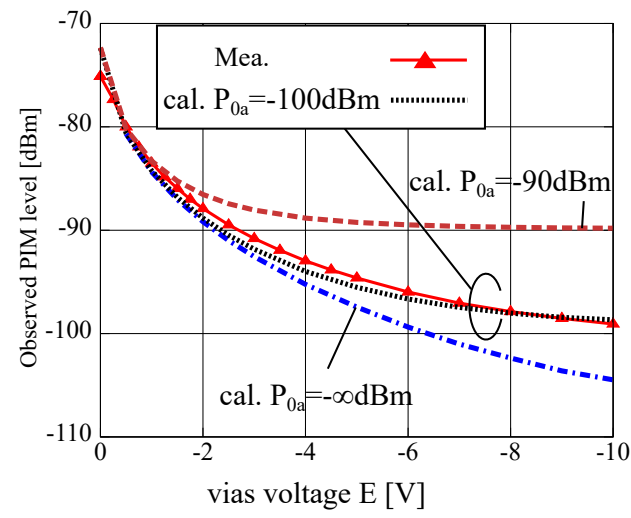

Fig. 3. Measured and calculated PIM characteristics of variable non-linear part for reverse bias voltage. $\left(P_{i n}^{\prime}=23 \mathrm{dBm} /\right.$ tone $)$

\section{EFFECTIVENESS EVALUATION OF DYNAMIC RESIDUAL NOISE IDENTIFICATION METHOD USING VARIABLE LOW-PIM TERMINATION}

Fig. 4 shows the configuration of the measurement system. The two kinds of known PIM source are used as residual noise to be identified herein, and which are referred to PIM-sourceA(high) and PIM-source-B(low), respectively. At this time, it is assumed that the residual noise of about $-100 \mathrm{dBm}$ for the applied power of $P_{i n}^{\prime \prime}=43 \mathrm{dBm} /$ tone is given to be identified. In this case, it is calculated by the equation (3) that a linear attenuator of about $-20 \mathrm{~dB}$ is required. The linear attenuator used in this letter is designed by UT141 of about $40 \mathrm{~m}$ so that its loss will be about $-20 \mathrm{~dB}$ at the test frequency $f_{1}, f_{2}, f_{\text {pim }}$.

Table I shows the transmission coefficient of the linear attenuator measured by a vector network analyzer at test frequencies $f_{1}, f_{2}, f_{\text {pim }}$. It is confirmed to be fully ideal for the requirement.

TABLE I

MEASURED TRANSMISSION COEFFICIENTS $\left(S_{21}\right)$ OF LINEAR ATTENUATOR

\begin{tabular}{c|ccc}
\hline & $f_{1}$ & $f_{2}$ & $f_{\text {pim }}$ \\
\hline \hline$S_{21}$ & $-20.4 \mathrm{~dB}$ & $-21.2 \mathrm{~dB}$ & $-19.6 \mathrm{~dB}$ \\
\hline
\end{tabular}

Fig. 5 shows the measurement results by using proposed residual noise identification method. The horizontal axis shows the bias voltage applied to the variable IM-source. The the dash-dot line show the generated PIM level by PIM-source-A and -B. They are employed as models of a residual noise to be identified, because each PIM level were measured in advance. Input power is $P_{i n}^{\prime \prime}=43 \mathrm{dBm} /$ tone. The solid line shows the observed PIM level of the termination itself, and the one with blue squares shows the calculated result by the equation (2).
It is confirmed that the observed PIM level saturates to the PIM level of PIM-source-A and -B as the reverse bias voltage increases. It indicates that the system residual noise can be identified by using the proposed variable low-PIM termination even for the practical power such as $43 \mathrm{dBm} /$ tone.

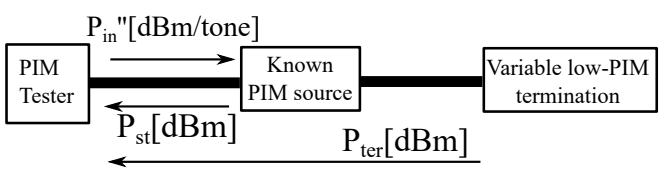

Fig. 4. Configuration of measurement system in residual noise identification

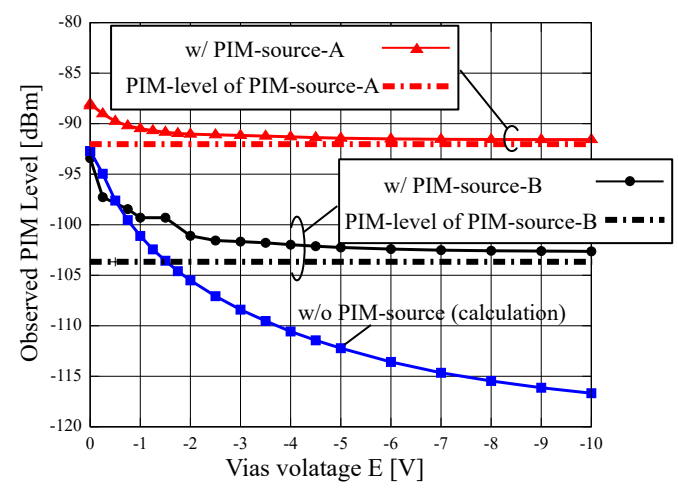

Fig. 5. Residual noise identification using variable low-PIM termination. $\left(P_{i n}^{\prime \prime}=43 \mathrm{dBm} /\right.$ tone $)$

\section{CONCLUSION}

In this letter, a novel variable low-PIM termination is proposed to identify the system residual noise of PIM measurement system. Since it was composed of an active element producing voltage-controlled IM, a fixed attenuator, and a linear attenuator; the produced IM level of the termination was always known and tunable. The method to consider the PIM level caused by the fixed attenuator was also described in order not to limit the tunable range of produced IM. As a consequence, it was shown that the proposed termination enabled to identify the residual noise in PIM tester by observing the saturated PIM level for the vias voltage sweep. The validity of proposed method was experimentally confirmed by evaluating the 3rd-order PIM in the $2 \mathrm{GHz}$ band.

\section{REFERENCES}

[1] Y. Patenaude, J. Dallaire, F. Menard, and S. Richard, "Antenna PIM measurements and associated test facilities," proc. IEEE AP-S International Symposium 2001(Digest), pp.620-623, Jul. 2001.

[2] J.R. Wilkerson, I.M. Kilgore, K.G. Gard, and M.B. Steer, "Passive Intermodulation Distortion in Antennas," IEEE Trans. Antennas Propag., vol. 63, issue 2, pp. 474-482, Feb. 2015.

[3] J. Sanford, "Passive Intermodulation Considerations in Antenna Design," proc. IEEE AP-S International Symposium 1993, pp.1651-1654, Jun. 1993.

[4] S. Chou, H. Chou, and L. Kuo, "Potential Causes of PIM Problems in the LTE," 2016 International Symposium on Antennas and Propagation (ISAP), pp1080-1081, Oct. 2016

[5] Y. Yamamoto, and N. Kuga, "PIM characteristics of resistive loads," proc. 2005 Asia-Pacific Microwave Conference (APMC), Dec. 2005. 
[6] RF connectors, connector cable assemblies and cables-intermodulation level measurement, IEC standard 62037-1, Jul. 2012.

[7] N. Kuga, and K. Nemoto, "Low-PIM termination design using a resistive termination," IEICE Electron. Expr., vol.7, no.3, pp.222-227, Feb. 2010

[8] J. Henrie, A. Christianson, and W.J. Chappell, "Prediction of passive intermodulation from coaxial connectors in microwave networks," IEEE Trans. Microw.Theory Tech., vol.56, issue 1, pp.209-216, Jan. 2008. 